\title{
Toroidal magnetic states in molecular wheels: Interplay between isotropic exchange interactions and local magnetic anisotropy
}

\author{
Alessandro Soncini ${ }^{1,2, *}$ and Liviu F. Chibotaru ${ }^{2}$ \\ ${ }^{1}$ Centre for Theoretical and Computational Chemistry (CTCC), University of Oslo, P.O. Box 1033 Blindern, N-0315 Oslo, Norway \\ ${ }^{2}$ Afdeling Kwantumchemie, Katholieke Universiteit Leuven, Celestijnenlaan 200F, B-3001 Heverlee, Belgium
}

(Received 2 April 2008; published 19 June 2008)

\begin{abstract}
It is shown that magnetic quantum states characterized by a toroidal moment of the same order of magnitude as the routinely measured molecular magnetization can arise in molecular wheels from the interplay between isotropic exchange and on-site magnetic anisotropy. They represent the first example of noncollinear molecular Néel states whose coupling can be strongly quenched as function of the orientation of the local anisotropy axes.

DOI: 10.1103/PhysRevB.77.220406

PACS number(s): 75.50.Xx, 75.10.Jm, 75.30.Cr, 75.30.Gw
\end{abstract}

Nanostructures exhibiting a curling in-plane (vortex) magnetic configuration are extensively investigated as novel materials for magnetic data storage technology. ${ }^{1}$ Recent advancements in the field have been achieved via the experimental characterization of vortex states in iron nanoislands, ${ }^{2}$ and of a ferrotoroidic magnetic phase in the oxide compound $\mathrm{LiCoPO}_{4} \cdot{ }^{3}$ Unusual transitions to ordered phases characterized by an electric toroid moment have been predicted in nanodisks and nanorods. ${ }^{4}$ Toroidal configurations have insofar been identified solely as thermodynamic phases, and as such their application is limited to information storage within classical binary states. ${ }^{5}$ The identification of a quantum toroidal magnetic configuration in single molecules would extend the application of vortex states to the domain of quantum computation. Although toroidal moments in molecules have been investigated as specific magnetic response properties leading to chiral discrimination, ${ }^{6}$ to date the predicted effects are too small to be observed.

Molecular wheels have been proposed as promising candidates for the implementation of spin qubits. ${ }^{7}$ The dephasing time of the associated spin states is determined by three main causes: phonons, nuclear spins, and intermolecular dipolar coupling. ${ }^{8}$ Whereas it has been shown that coupling to phonons and electron spin coupling to proton spins can be efficiently reduced at low temperatures and via deuteration, ${ }^{9}$ dipolar coupling between neighboring molecules remains a drawback that can solely be addressed by sparse packing of the molecular wheels. In this respect, provided it is possible to devise an efficient way to address their states, magnetic toroidal qubits would in principle offer a route to closer packing, as their interaction with $\boldsymbol{\nabla} \times \mathbf{B}$ decays much faster with intercluster separation than dipolar interactions. However, all studies reported insofar on molecular nanomagnets always assume that on-site magnetic anisotropies are characterized by a collinear arrangement, thereby excluding from the very start the possibility for the existence of noncollinear magnetic states, such as the above-mentioned vortex states. In this work we show that it is possible to prepare stable toroidal magnetic states in molecular wheels, arising solely from the interplay between isotropic exchange interaction and local magnetic anisotropy.

The low-lying states of a wheel can be modeled by the following effective spin Hamiltonian: ${ }^{10}$

$$
\mathrm{H}=-J \sum_{i} \mathbf{s}_{i} \cdot \mathbf{s}_{i+1}+\sum_{i} D_{i} s_{z, i}^{2}
$$

where $J$ is the exchange coupling constant and $D_{i}$ is the single-ion axial zero-field splitting (ZFS) parameter. A common assumption is to consider the local anisotropy axes all parallel to the wheel uniaxis, so that the spin operators appearing in Eq. (1) are all defined with respect to a common reference frame. However, this is hardly ever the situation met in real wheels. ${ }^{11}$ Furthermore, in order to explore toroidal magnetic states the "collinear" hypothesis must be abandoned. Let us then consider plausible orientations of the local axes, complying with the often encountered $C_{N / 2}$ or $C_{N}$ rotational symmetries of wheels; the local anisotropy axes are assumed to be in planes tangential to the wheel's circumference, and characterized by either (i) an alternating tilting angle $\pm \theta$ with respect to the main symmetry axis complying with $C_{N / 2}$ symmetry (alternating configuration, see Fig. 1), or (ii) the same tilting angle $+\theta$ for all sites, complying with $C_{N}$ symmetry (parallel configuration).

The pure spin toroidal moment operator is defined as $\tau$ $=g \mu_{B} \Sigma_{i}^{N} \mathbf{r}_{\mathbf{i}} \times \mathbf{s}_{i}$, where $\mu_{B}$ is the Bohr magneton and $g$ the isotropic $g$ factor. Ideal toroidal states would have to be char-
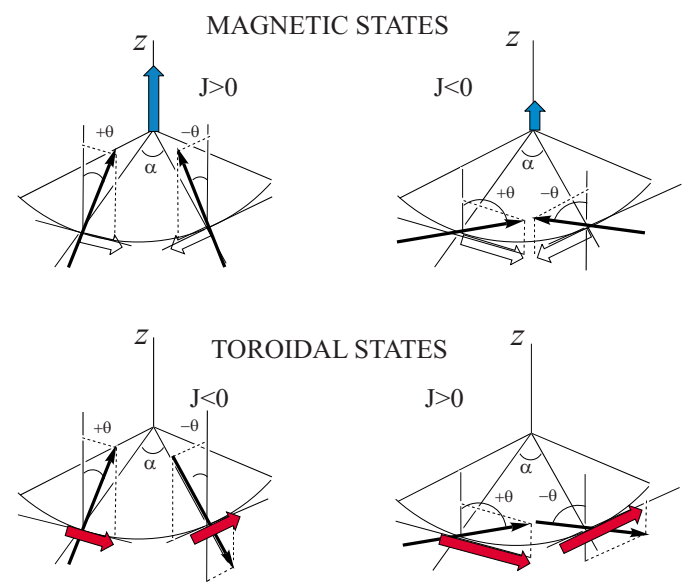

FIG. 1. (Color online) The interplay between tilting of local easy axes $(\theta)$ and (anti)ferromagnetic isotropic exchange $J$ in molecular wheels leads either to magnetic quantum states (top) or to toroidal quantum states (bottom). 
acterized by an exact toroidal quantum number. It can nevertheless be shown that both for alternating and for parallel configurations $\left[\tau_{z}, \mathrm{H}\right] \neq 0$. However, we show here that a realistic route to magnetic vortex states can be pursued by considering the highly anisotropic limit of Eq. (1). In the limit $\left|D_{i}\right| \gg|J|$ characterizing molecular wheels made of, e.g., $\mathrm{Co}(\mathrm{II})$ ions, ${ }^{12}$ a convenient basis to analyze the solutions of Eq. (1) for $N$ equivalent magnetic centers with spin $s$, and spin projection $m_{i}$ along the local easy axis, is given by the direct product of the single-ion spin states. Let us consider the alternating configuration (Fig. 1), arguably the most commonly encountered. Written in local spin coordinates, we can partition Eq. (1) as $H=H_{\mathrm{ZFS}}+H_{\mathrm{exch}}^{0}+H_{\mathrm{exch}}^{1}$, where $H_{\mathrm{ZFS}}$ $=D \Sigma_{i} s_{z, i}^{2}, \quad H_{\text {exch }}^{0}=-J^{\prime} \sum_{i} s_{z, i} s_{z, i+1}$ is an Ising-type exchange Hamiltonian. The Ising coupling constant is given by $J^{\prime}$ $=J\left(\cos ^{2} \theta-\cos \alpha \sin ^{2} \theta\right)$, where angles $\alpha$ and $\theta$ are defined as in Fig. 1. The basis functions $\left|m_{1}, m_{2}, \ldots, m_{N}\right\rangle$ are eigenfunctions of the zeroth-order Hamiltonian $H_{\mathrm{ZFS}}+H_{\text {exch }}^{0}$ with eigenvalues $E_{\mathbf{m}}=D \sum_{i} m_{i}^{2}-J^{\prime} \sum_{i} m_{i} m_{i+1}$. The ground state can then be described in terms of the $2^{N}$-degenerate ground ZFS multiplet associated to the products of local spin states with maximal positive or negative projections along the local (tilted) anisotropy axes, splitted into $(N / 2)+1$ sets of degenerate levels equally spaced by energy gaps of $4 J s^{2}$ by the Ising exchange.

The resulting zeroth-order ground state is always doubly degenerate. In particular, if $J>0$ (ferromagnetic coupling) and $\theta<\theta_{0}$ or $J<0$ (antiferromagnetic coupling) and $\theta>\theta_{0}$, where $\theta_{0}=\arctan (1 / \sqrt{\cos \alpha})$ (top row of Fig. 1), the ground state can be described in terms of the ferromagnetic Ising doublet $|+s+s \cdots+s\rangle$ and $|-s-s \cdots-s\rangle$. These states are magnetic as they possess a magnetic moment along the wheel uniaxis given by $M_{z}= \pm g \mu_{B} N s \cos \theta$, which is also a good quantum number. On the other hand, if $J<0$ and $\theta<\theta_{0}$ or $J>0$ and $\theta>\theta_{0}$ (bottom row of Fig. 1), the ground state of the wheel within the Ising-exchange picture is described by the Néel doublet $|+s-s \cdots-s\rangle$ and $|-s+s \cdots+s\rangle$. These are toroidal states, as for any $\theta \neq 0^{\circ}$ they are characterized by (i) $\mathbf{M}=0$ (ii) and an origin-invariant toroidal moment (good quantum number) aligned along the wheel's uniaxis, given by $\tau_{z}= \pm g \mu_{B} R N s \sin \theta$ ( $R$ is the wheel's radius). Within the lowest ZFS multiplet, these states represent exact solutions, since for $s>1 / 2$ the operator $H_{\mathrm{exch}}^{1}$ is only able to couple the ground state doublet with excited ZFS multiplets, accessible via an energy gap of the order of $D$.

How does this picture survive beyond the simple Ising approximation? If $\theta=0^{\circ}$, the non-Ising part of the exchange Hamiltonian is proportional to $\sum_{i}\left(s_{+, i} s_{-, i+1}+s_{-, i} s_{+, i+1}\right)$, so that (i) the ferromagnetic Ising states are never coupled and thus remain degenerate (ii) the Néel states can be coupled in high order of perturbation theory (PT), a fact that gives rise to the splitting of the degenerate doublet, and to tunneling phenomena of the Néel vector. ${ }^{13}$ However, when $\theta \neq 0^{\circ}$, the Ising degeneracy can be removed both for ferromagnetic states and for Néel (toroidal) states, in both alternating and parallel configurations. This is most easily seen, in, e.g., the alternating case, by decomposing the perturbation $H_{\text {exch }}^{1}$ into singlesite excitations $H^{1 s}$ (changes the spin on one site only), and nearest-neighbors double-excitations $H^{1 d}$ (changes the spin on nearest-neighbor sites). In order to couple the two com- ponents of the Ising doublet it is necessary for the perturbation to invert the spin on each site, so that the simultaneous excitation of two nearest-neighbor sites give rise to tunneling processes in Nsth order of PT, whereas single-site excitations give rise to much weaker mechanisms whose PT order is at least twice as large. Considering only $H^{1 d}$, the coupling Hamiltonian is written in terms of local spin coordinates as

$$
\begin{aligned}
H^{1 d}= & \frac{\sin ^{2} \theta(\cos \alpha-1)}{4} \sum_{r}\left(s_{+}^{r} s_{+}^{r+1}+s_{-}^{r} s_{-}^{r+1}\right) \\
& +\frac{2 \cos ^{2} \theta \cos \alpha+\sin ^{2} \theta(\cos \alpha-1)}{4} \sum_{r}\left(s_{+}^{r} s_{-}^{r+1}+s_{-}^{r} s_{+}^{r+1}\right) \\
& -\frac{i \cos \theta \sin \alpha}{2} \sum_{r}\left(s_{+}^{r} s_{-}^{r+1}-s_{-}^{r} s_{+}^{r+1}\right) .
\end{aligned}
$$

From Eq. (2) it is evident that both ferromagnetic and Néel states degeneracy can be removed when $\theta \neq 0^{\circ}$ : the former via mechanisms based on $\Sigma_{r}\left(s_{+}^{r} s_{+}^{r+1}+s_{-}^{r} s_{-}^{r+1}\right)$, the latter via mechanisms based on $\sum_{r}\left(s_{+}^{r} s_{-}^{r+1}+s_{-}^{r} s_{+}^{r+1}\right)$ and $\sum_{r}\left(s_{+}^{r} s_{-}^{r+1}\right.$ $\left.-s_{-}^{r} s_{+}^{r+1}\right)$. Nevertheless, the realization of molecular toroidal (or ferromagnetic) states can be expected to be feasible on the basis of three considerations. First, beyond some threshold value for $|D / J|$ the higher ZFS multiplets will be weakly coupled to the ground state doublet. Second, the angular dependence in Eq. (2) can be expected to lead to a set of orientations of the tilting axes for which the relevant coupling mechanisms will be vanishingly small. Finally, since the lowest order of PT characterizing the coupling process is $N s$, the higher $N$ and $s$ the weaker the splitting produced.

In order to explore these ideas quantitatively and to determine the range of parameters needed for the realization of toroidal states in molecular wheels, we proceed by calculating the exact eigenstates of Eq. (1). We report here the results of the full diagonalization for the alternating configuration case, with $N=6$ and $s=1$. Figure 2 shows a plot of the energy gap between the ground and the first excited state (i.e., the exact splitting of the ground state Ising doublet) as function of the tilting angle $\theta$ and the $|D / J|$ ratio. As expected the gap decreases with increasing $|D / J|$. However, the decrease rate shows markedly different features as function of the angle $\theta$. For $N=6$ we have $\theta_{0}=54.7^{\circ}$. From the plot we can see that when $\theta<\theta_{0}$ (toroidal regime) the magnitude of the gap steadily decreases as function of $|D / J|$, with larger rate for smaller angles. The gap narrows with the slowest rate for $\theta$ $=\theta_{0}$, for which value of the tilting the gap remains larger than $0.1|J|$ even for $|D / J| \approx 10$. This implies that for $\theta$ $<\theta_{0}$ the smaller the angle the more stable is the degenerate toroidal ground state. However small angles imply small values of $\tau_{z}$. For $\theta>\theta_{0}$ the Ising ground state is ferromagnetic. In this case, although a coupling mechanism is active, it can be seen that the gap has a very sudden drop to about $10^{-4}|J|$ for $|D / J| \approx 5$.

Much more promising for the realization of toroidal states in molecular wheels is the case of ferromagnetic coupling. In Fig. 3 we report the plot of the corresponding Ising doublet splitting. In the strong exchange limit $(|D / J| \ll 1)$ the ground state is doubly degenerate for all angles $\theta<\theta_{0}$, as expected 


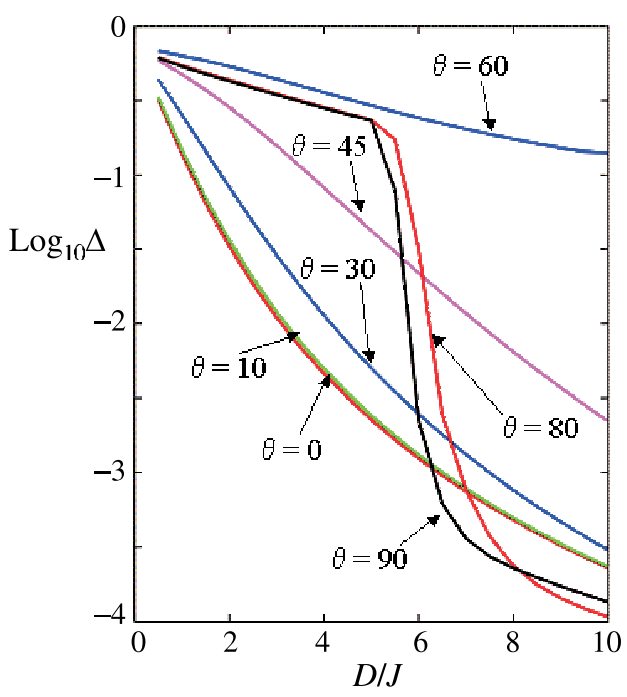

FIG. 2. (Color online) Logarithmic plot (base 10) of the Ising doublet splitting $(\Delta)$ obtained by exact diagonalization of Eq. (1) in six-center antiferromagnetic wheels with $s=1$ as function of the local tilting angle $\theta$ (alternating local axes) and $|D / J|$.

by straightforward projection of the ZFS Hamiltonian within the ground state exchange multiplet. Even for larger $|D / J|$, when $\theta<40^{\circ}$, the wheel's states can be described in terms of a degenerate ferromagnetic Ising doublet, despite the fact that the degeneracy is exact only for $\theta=0^{\circ}$. The relevant coupling mechanism becomes efficient only for $\theta>40^{\circ}$, making the gap larger up to a maximal value. Then the gap decays with increasing $|D / J|$. For $\theta<\theta_{0}$, the larger the angle, the slower the decrease, reaching a minimal decreasing rate for $\theta=\theta_{0}$. For $\theta>\theta_{0}$ the ground state is described in terms of toroidal Néel states, coupled in high order PT via the relevant mechanisms in Eq. (2). For angles $\theta>70^{\circ}$ and already for $|D / J| \approx 4$ the splitting of the Néel states drops very rapidly to values smaller than $10^{-5}|J|$, so that these states can be

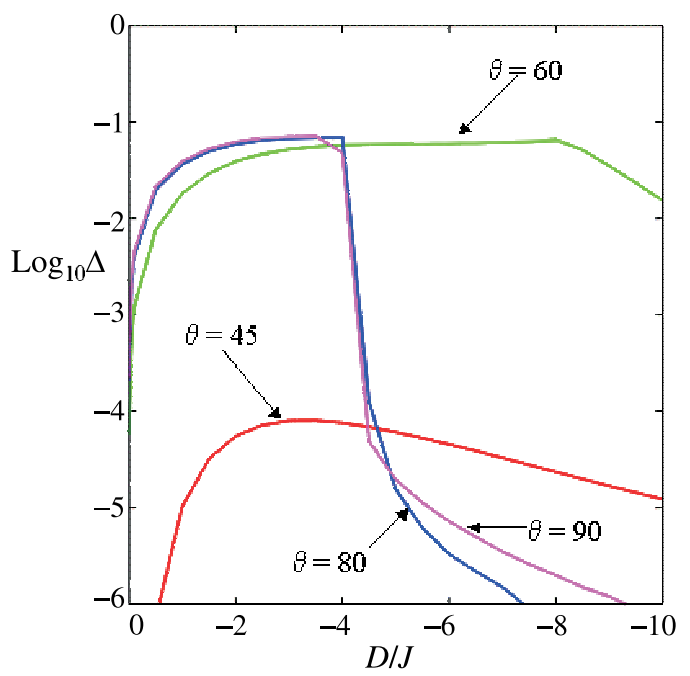

FIG. 3. (Color online) Logarithmic plot (base 10) of the Ising doublet splitting $\Delta$ obtained by exact diagonalization of Eq. (1) in six-center ferromagnetic wheels with $s=1$ as function of the local tilting angle $\theta$ (alternating local axes) and $|D / J|$.



FIG. 4. (Color online) Toroidal $\left(\tau_{z}\right)$ and magnetic $\left(M_{z}\right)$ moments in six-center wheels $(s=1)$, plotted as functions of the tilting angle $\theta$ for $|D / J|=10$, for both $J>0$ and $J<0$.

considered effectively degenerate. Furthermore, for large values of the angle $\theta$ the estimate of $\tau_{z}$ based on the Ising model reaches its maximal magnitude, and the gap between Néel states its minimal value. Hence, in the ferromagnetic alternating configuration with $\theta>\theta_{0}$, already for $|D / J| \approx 4$, we can expect the doubly degenerate ground state to be appropriately described in terms of a toroidal quantum number. Note that these results are not expected to depend qualitatively on the details of the exchange Hamiltonian in Eq. (1), chosen here isotropic for simplicity, given the large ratio between anisotropy and exchange parameters.

The evolution of $\tau_{z}$ and $M_{z}$ as functions of $\theta$ has been calculated using the exact ground state spin functions. ${ }^{14}$ The results are reported in Fig. 4 for $|D / J|=10$. It can be clearly seen that the greater stability of the degenerate ground state (both toroidal and magnetic) achieved in the ferromagnetic coupling case results in larger $M_{z}$ and $\tau_{z}$ values for a wider range of angles. For $|D / J|=10$ the maximal value of $\tau_{z}(\theta$ $=90^{\circ}$ ) is already equal to the value estimated on the basis of the unperturbed Ising model $\left(\tau_{z} / R=6 g \mu_{B}\right)$. The reason for the efficiency of ferromagnetic coupling can be qualitatively rationalized by plotting the $N$ sth power (sixth in this case) of the angular factor multiplying the relevant operators in Eq. (2). The plots are reported in Fig. 5. Interestingly, it can be seen that the range of $\theta$ values for which toroidal states coupling is active ( $\operatorname{small} \theta$ ), and the range of $\theta$ values for which coupling between ferromagnetic states is most efficient (large $\theta$ ), almost do not overlap. Hence, in the antiferromagnetic (ferromagnetic) case there occurs a perfect match (mismatch) between the (toroidal or ferromagnetic) nature of the ground state doublet, and the ability of the transversal 


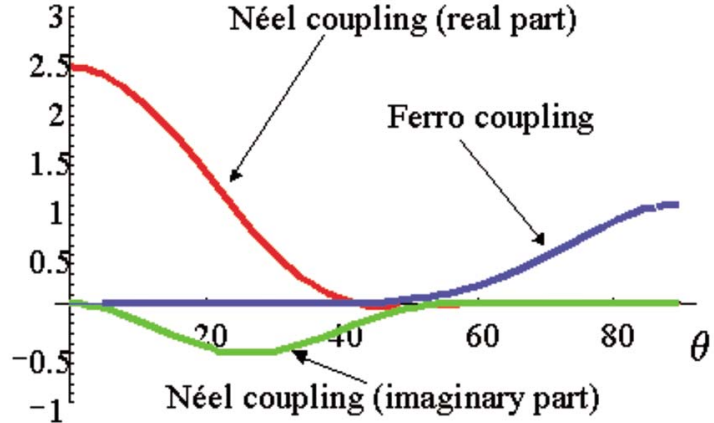

FIG. 5. (Color online) Angular dependence of the dominant coupling mechanisms in sixth-order PT between Néel and Ising states, in a six-center wheel with local $s=1$.

Hamiltonian to remove the degeneracy. Ferromagnetic wheels achieve maximal magnetization for small angles (Fig. 4) because the dominant mechanism able to remove degeneracy is vanishingly small for $\theta<45^{\circ}$ (Fig. 5). Likewise, for large angles, a very large $\tau_{z}$ value is achieved because the mechanisms able to disrupt the Néel states degeneracy are almost inactive for $\theta>40^{\circ}$. The same arguments rationalize the smaller values of $\tau_{z}$ and $M_{z}$ when $J<0$.

The parallel configuration can be discussed in similar terms, although now the zeroth-order ground state of antifer- romagnetic (ferromagnetic) wheels is described by Néel (Ising-ferromagnetic) doublet for any value of $\theta$, so that toroidal configurations can only be realized for ferromagnetic molecules. The recently reported measurements of vanishing low-temperature and/or low-field response to uniform magnetic fields of molecular wheels made up of dysprosium triangles ${ }^{15}$ are unprecedented results in systems with an odd number of unpaired electrons, and $a b$ initio investigations have shown that they can be explained in terms of purely toroidal magnetic states with vanishing magnetic dipole moment. ${ }^{16}$ The ferromagnetic cobalt wheel recently reported in Ref. 12 represents another promising test case for the experimental detection of $\tau_{z}$.

In conclusion, we have provided a characterization of molecular noncollinear magnetic states by introducing the idea of toroidal magnetic states in molecular wheels, and quantitatively identified the optimal conditions for their realization. These findings represent an advancement in the understanding of molecular magnetic nanoclusters in the strong anisotropy regime, and can provide the basis for further research on the identification of novel strategies for the implementation of molecular qubits not based on conventional spin states.

A.S. acknowledges the Francqui Foundation, the Flemish Science Foundation (FWO), and the University of Leuven. *alessandro.soncini@kjemi.uio.no

${ }^{1}$ K. Bussmann, G. A. Prinz, S. F. Cheng, and D. Wang, Appl. Phys. Lett. 75, 2476 (1999); W. Eerenstein, N. D. Mathur, and J. F. Scott, Nature (London) 442, 759 (2006).

${ }^{2}$ A. Wachowiak, J. Wiebe, M. Bode, O. Pietzsch, M. Morgenstern, and R. Wiesendanger, Science 298, 577 (2002).

${ }^{3}$ B. B. Van Aken, J. P. Rivera, H. Schmid, and M. Fiebig, Nature (London) 449, 702 (2007).

${ }^{4}$ I. I. Naumov, L. Bellaiche, and H. X. Fu, Nature (London) 432, 737 (2004)

${ }^{5}$ M. Kläui, C. A. F. Vaz, J. A. C. Bland, T. L. Monchesky, J. Unguris, E. Bauer, S. Cherifi, S. Heun, A. Locatelli, L. J. Heyderman, and Z. Cui, Phys. Rev. B 68, 134426 (2003).

${ }^{6}$ I. B. Khriplovich and M. E. Pospelov, Z. Phys. D: At., Mol. Clusters 17, 81 (1990); R. R. Lewis, Phys. Rev. A 49, 3376 (1994); A. Ceulemans, L. F. Chibotaru, and P. W. Fowler, Phys. Rev. Lett. 80, 1861 (1998). F. Faglioni, A. Ligabue, S. Pelloni, A. Soncini, and P. Lazzeretti, Chem. Phys. 304, 289 (2004).

${ }^{7}$ F. Troiani, A. Ghirri, M. Affronte, S. Carretta, P. Santini, G. Amoretti, S. Piligkos, G. Timco, and R. E. P. Winpenny, Phys. Rev. Lett. 94, 207208 (2005); S. Carretta, P. Santini, G. Amoretti, F. Troiani, and M. Affronte, Phys. Rev. B 76, 024408 (2007).

${ }^{8}$ A. Morello, P. C. E. Stamp, and I. S. Tupitsyn, Phys. Rev. Lett. 97, 207206 (2006).

${ }^{9}$ A. Ardavan, O. Rival, J. J. L. Morton, S. J. Blundell, A. M.
Tyryshkin, G. A. Timco, and R. E. P. Winpenny, Phys. Rev. Lett. 98, 057201 (2007); W. Wernsdorfer, Nat. Mater. 6, 174 (2007).

${ }^{10}$ A. Bencini and D. Gatteschi, EPR of Exchange Coupled Systems (Springer, Berlin, 1990).

${ }^{11}$ M. Affronte, S. Carretta, G. A. Timco, and R. E. P. Winpenny, Chem. Commun. (Cambridge) 2007, 1789; G. A. Timco, A. S. Batsanov, F. K. Larsen, C. A. Muryn, J. Overgaard, S. J. Teat, and R. E. P. Winpenny, ibid. 2005, 3649.

${ }^{12}$ J. K. Beattie, T. W. Hambley, J. A. Klepetko, A. F. Masters, and P. Turner, Chem. Commun. (Cambridge) 1998, 45; E. K. Brechin, O. Cador, A. Caneschi, C. Cadiou, S. G. Harris, S. Parsons, M. Vonci, and R. E. P. Winpenny, ibid. 2002, 1860; M. Moragues-Canovás, C. E. Talbot-Eeckelaers, L. Catala, F. Lloret, W. Wernsdorfer, E. K. Brechin, and T. Mallah, Inorg. Chem. 45, 7038 (2006).

${ }^{13}$ O. Waldmann, C. Dobe, H. Mutka, A. Furrer, and H. U. Gudel, Phys. Rev. Lett. 95, 057202 (2005).

${ }^{14}$ Physically, the saturated values of $\tau_{z}$ and $M_{z}$ are obtained by applying stabilizing fields, large enough to overcome the splitting of the ground Ising doublet.

${ }^{15}$ J. Tang, I. Hewitt, N. T. Madhu, G. Chastanet, W. Wernsdorfer, C. E. Anson, C. Benelli, R. Sessoli, and A. K. Powell, Angew. Chem., Int. Ed. 45, 1729 (2006).

${ }^{16}$ L. F. Chibotaru, L. Ungur, and A. Soncini, Angew. Chem., Int. Ed. 47, 4126 (2008). 